\title{
The Arbitrary Trajectory Quantization Method
}

\author{
Debabrata Biswas \\ Theoretical Physics Division \\ Bhabha Atomic Research Centre \\ Mumbai 400 085, INDIA
}

(November 21, 2018)

\begin{abstract}
The arbitrary trajectory quantization method (ATQM) is a time dependent approach to quasiclassical quantization based on the approximate dual relationship that exists between the quantum energy spectra and classical periodic orbits. It has recently been shown however, that, for polygonal billiards, the periodicity criterion must be relaxed to include closed almost-periodic (CAP) orbit families in this relationship. In light of this result, we reinvestigate the ATQM and show that at finite energies, a smoothened quasiclassical kernel corresponds to the modified formula that includes CAP families while the $\delta$ function kernel corresponding to the periodic orbit formula is recovered as $E \rightarrow \infty$. Several clarifications are also provided.
\end{abstract}

Published in Phys. Rev. E 63, 016213, (C)The American Physical Society

\section{INTRODUCTION}

Semiclassical quantization methods developed over the past three decades generally rely on the energy domain approach. The spectral density, $\rho(E)=\sum_{n} \delta\left(E-E_{n}\right)$ is thus expressed in terms of periodic orbits of the underlying classical dynamics and this is commonly referred to as the Gutzwiller trace formula [1]. The crossover from the time domain to the energy domain and the trace formula necessitates several approximations in the form of stationary phase integrations and one of these selects periodic orbits as the sole classical ingredient. The recipe works well in most cases since closed non-periodic orbits contribute with a lesser weight and can be included only as a correction. There are instances however when closed non-periodic orbits contribute with weights comparable to periodic orbits and the "periodicity criterion" must then be relaxed to accommodate them [2,3]. Polygonal billiards provide such an example and perhaps hold the key to generic intermittent behaviour. In these systems, a slight change in the internal angles results in the destruction of periodic orbit families thereby giving rise to closed almost-periodic (CAP) families of orbits [4]. Since this is true for each periodic family in every neighbouring polygon, CAP families actually outnumber periodic families of any given polygon. Further, they contribute with weights comparable to periodic families in the semiclassical trace formula and are hence indispensable for semiclassical quantization. This makes the energy domain approach rather cumbersome to implement and a time domain approach is thus preferable.

For polygonal billiards, there are two available approaches in the time domain. The first of these developed by Heller and Tomsovic [5] relies on a semiclassical construction of the time dependent propagator to evaluate the auto-correlation function, $C(t)=<\psi \mid \psi(t)>$ where $\psi(\mathbf{q}, t)=<\mathbf{q} \mid \psi(t)>=\sum_{n} c_{n} \phi_{n}(\mathbf{q}) e^{-i E_{n} t / \hbar} \simeq$ $\int K_{s . c}\left(\mathbf{q}, \mathbf{q}^{\prime}, t\right) \psi\left(\mathbf{q}^{\prime}, 0\right) d \mathbf{q}^{\prime}$. Here $\left\{\phi_{n}\right\},\left\{E_{n}\right\}$ and $\left\{c_{n}\right\}$ are the eigenfunctions, eigenvalues and expansion coefficients respectively while $K_{s . c}\left(\mathbf{q}, \mathbf{q}^{\prime}, t\right)$ is the semiclassical propagator constructed using classical trajectories joining a pair of points $\left(\mathbf{q}, \mathbf{q}^{\prime}\right)$ at each time $t$. A power spectrum of $\psi(\mathbf{q}, t)$ thus yields the quantum eigenvalues. The method has been successfully applied to the stadium billiard but to the best of our knowledge, it has not been used for quantizing polygonal billiards.

The second approach [6, i] is simpler to adopt and relies on classical propagation (or its quasiclassical [8] adaptation where necessary). In case of a polygon, it involves shooting arbitrary trajectories in various directions from a point (call it $\mathbf{q}^{\prime}$ - see fig. 1, left) and at each time step, recording the (weighted) fraction, $F(t)$ of trajectories that are in an $\epsilon$ neighbourhood of a point $\mathbf{q}$. The peaks in the power spectrum of $F(t)$ are then related to the quantum eigenvalues.
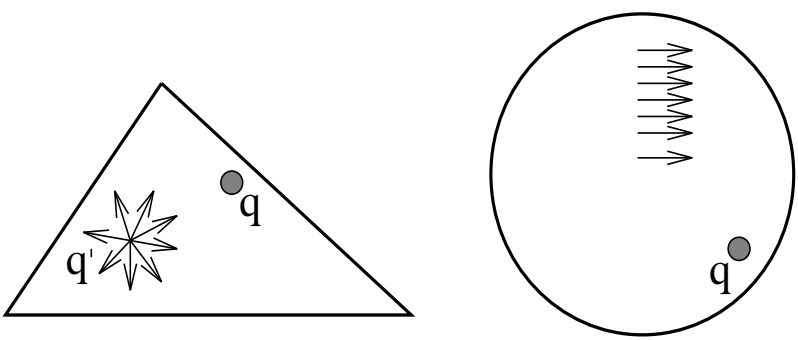

FIG. 1. The arbitrary trajectory quantization recipe. In case of the polygon, trajectories with different linear momentum span the entire constant energy surface. In case of the circle, this is achieved by shooting trajectories with different angular momentum. In both cases, the (weighted) fraction of trajectories in the cell around $\mathbf{q}$ is recorded.

Apart from the simplicity, the arbitrary trajectory quantization method (ATQM) (see [6]) is perhaps the only semiclassical scheme that has been successful in determining the first few quantal energy levels of generic polygonal billiards [9]. However, the theory as presented in [6] leaves a number of questions unanswered. For one, the role of the $\epsilon$ neighbourhood is unclear. Besides, as the theory uses periodic orbit quantization to relate the peaks in the power spectrum of $F(t)$ with the quantum eigenenergies, the role of CAP trajectories must be clarified. We shall thus reinvestigate ATQM from this view- 
point and understand why it works.

The plan of the paper is as follows. In section II, we shall recapitulate the existing work on the arbitrary trajectory quantization method. The modifications that we shall carry out to account for the inclusion of closed almost-periodic orbits can be found in section III] and this constitutes the main part of this paper. Finally, a discussion on errors and a summary of our results can be found in section $[\mathrm{IV}$.

\section{ARBITRARY TRAJECTORY QUANTIZATION : A REVIEW}

The arbitrary trajectory quantization method relies on a suitable (quasiclassical) adaptation of the classical evolution operator that propagates a density under a flow that we denote by $q^{t}$. For polygonal billiards, the flow occurs on an invariant surface that is 2 -dimensional and characterized by the two constants of motion, $\{E, \varphi\}$ where $E$ is the energy and $\varphi$ denotes the second constant. The surface has the topology of a sphere with $g$ (called the genus) holes where $g$ can be determined from the internal angles of the polygon. The motion on the invariant surface can alternately be viewed on a singly connected surface obtained by executing $2 g$ cuts and with edges appropriately identified.

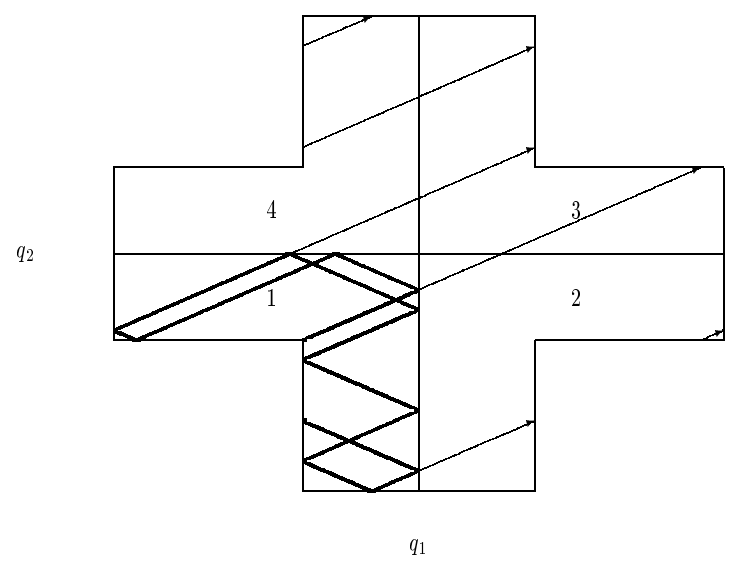

FIG. 2. The singly connected region for an L-shaped billiard consists of four copies with edges appropriately identified. A trajectory originating near the $3 \pi / 2$ vertex in 1 is plotted in configuration space using bold lines and the corresponding unfolded trajectory is also shown. The latter consists of parallel segments and the trajectory can be parameterized by the angle $\varphi$ that it makes for example with the $q_{1}$ axis.

As a trivial example, consider the rectangular billiard. The singly connected region is a larger rectangle consisting of four copies corresponding to the four directions that a trajectory can have and these can be glued appropriately to form a torus. As a non-trivial example, consider the L-shaped billiard of fig. 2 which is pseudo- integrable with its invariant surface having, $g=2$. Alternately, the surface can be represented by a singly connected region in the plane and consists of four copies corresponding to the four possible directions an orbit can have and these are glued appropriately. A trajectory in phase space thus consists of parallel segments at an angle $\varphi$ [10] measured for example with respect to one of the sides. It will be useful to note at this point that the same trajectory can also be represented by parallel segments at angles $\pi-\varphi, \pi+\varphi$ and $2 \pi-\varphi$. In general, the number of directions for representing a trajectory equals the number of copies, $N$, that constitute the invariant surface.

Before considering the question of quasiclassical quantization, we first introduce the appropriate classical evolution operator. For integrable systems, this is easily defined as

$$
\mathcal{L}^{t} \circ \phi\left(\theta_{1}, \theta_{2}\right)=\int d \theta_{1}^{\prime} d \theta_{2}^{\prime} \delta\left(\theta_{1}-\theta_{1}^{\prime t}\right) \delta\left(\theta_{2}-\theta_{2}^{\prime t}\right) \phi\left(\theta_{1}^{\prime}, \theta_{2}^{\prime}\right)
$$

where $\theta_{1}$ and $\theta_{2}$ are the angular coordinates on the torus and evolve in time as $\theta_{i}^{t}=\omega_{i}\left(I_{1}, I_{2}\right) t+\theta_{i}$ with $\omega_{i}=$ $\partial H\left(I_{1}, I_{2}\right) / \partial I_{i}$ and $I_{i}=\frac{1}{2 \pi} \oint_{\Gamma_{i}}$ p.dq. Here $\Gamma_{i}, i=1,2$ refer to the two irreducible circuits on the torus and $\mathbf{p}$ is the momentum conjugate to the coordinate $\mathbf{q}$.

On a general two dimensional invariant surface parameterized by $\varphi$, the classical propagator is expressed as [6]

$$
\mathcal{L}^{t}(\varphi) \circ \phi(\mathbf{q})=\int d \mathbf{q}^{\prime} \delta\left(\mathbf{q}-\mathbf{q}^{\prime t}(\varphi)\right) \phi\left(\mathbf{q}^{\prime}\right)
$$

where $\mathbf{q}$ refers to the position in the singly connected region (see fig. 2) and $\mathbf{q}^{\prime t}(\varphi)$ is the time evolution parameterized by $\varphi$ as described above. We denote by $\left\{\Lambda_{n}(t ; \varphi)\right\}$, the eigenvalues of $\mathcal{L}^{t}(\varphi)$ and from its multiplicative nature, it follows that $\Lambda_{n}(t ; \varphi)=e^{\lambda_{n}(\varphi) t}$. We are interested here in the form of $\lambda_{n}(\varphi)$ and to this end we shall evaluate

$$
\begin{aligned}
\int d \varphi \operatorname{Tr} \mathcal{L}^{t}(\varphi) & =\int d \varphi \sum e^{\lambda_{n}(\varphi) t} \\
& =\int d \varphi \int d \mathbf{q} \delta\left(\mathbf{q}-\mathbf{q}^{t}(\varphi)\right) .
\end{aligned}
$$

The delta function kernel ensures that the only orbits that contribute are ones that are periodic or close on the invariant surface after a time $t$. The $\mathbf{q}$ integrations are thus simpler to perform if we transform to a local coordinate system with one component parallel to the trajectory and the other perpendicular. Thus $\delta_{\|}\left(q_{\|}-q_{\|}^{t}\right)=\sum_{p} \sum_{r} \frac{1}{v} \delta\left(t-r T_{p}\right)$ where $v$ is the velocity, $T_{p}$ is the period of the orbit and $r$ is the repetition number. Similarly, for an orbit of period $r T_{p}$ parameterized by the angle $\varphi_{p}, \delta_{\perp}\left(q_{\perp}-q_{\perp}^{r T_{p}}\right)=\delta\left(\varphi-\varphi_{p}\right) /\left|\partial q_{\perp} / \partial \varphi\right|_{\varphi=\varphi_{p}}$ where $\left|\partial q_{\perp} / \partial \varphi\right|_{\varphi=\varphi_{p}}=r l_{p}$ for marginally unstable billiards. Putting these results together and noting that each periodic orbit occurs in general at $N_{p}$ different values of $\varphi$, we finally have 


$$
\begin{aligned}
\int d \varphi \sum e^{\lambda_{n}(\varphi) t} & =\sum_{p} \sum_{r=1}^{\infty} \frac{a_{p} N_{p}}{r l_{p}} \delta\left(l-r l_{p}\right) \\
& \simeq N \sum_{p} \sum_{r=1}^{\infty} \frac{a_{p}}{r l_{p}} \delta\left(l-r l_{p}\right)
\end{aligned}
$$

where $l=t v$ and the summation over $p$ refers to all primitive periodic orbit families with length $l_{p}$ and occupying an area $a_{p}$. Note that in Eq. (位), we have replaced $N_{p}$ by $N$ since for most long orbits, $N_{p} \simeq N$. Also, we have neglected the influence of isolated orbits to simply matters. We shall continue to make this approximation through the rest of this paper and justify its use at the end.

In some cases, it is possible to interpret the periodic orbit sum in Eq. (伍) starting with the semiclassical $(E \rightarrow$ $\infty)$ trace formula for marginally stable systems :

$$
\begin{aligned}
\rho(E) & \simeq \rho_{a v}(E) \\
& +\frac{1}{\sqrt{8 \pi^{3}}} \sum_{p} \sum_{r=1}^{\infty} \frac{a_{p}}{\sqrt{k r l_{p}}} \cos \left(k r l_{p}-\frac{\pi}{4}-r \mu_{p}\right) .
\end{aligned}
$$

Here $\rho_{a v}(E)$ refers to the average density of quantal eigenstates, $k=\sqrt{E}, l_{p}$ is the length of a primitive periodic orbit family, $\mu_{p}=\pi n_{p}+\nu_{p} \pi / 2, n_{p}$ the number of (phase-altering) bounces that it suffers at the boundary and $\nu_{p}$ the number of caustics encountered by the orbit. Note that in the Neumann case, $n_{p}=0$ since there is no phase loss on reflection while for polygonal billiards, $\nu_{p}=0$. For convenience, we have $\hbar=1, v=1$ and the mass $m=1 / 2$. Starting with the function

$$
\sum_{n} f\left(\sqrt{E_{n}} l\right) e^{-\beta E_{n}}=\int_{\Delta}^{\infty} d E f(\sqrt{E} l) e^{-\beta E} \sum_{n} \rho(E)
$$

where $f(x)=\sqrt{\frac{2}{\pi x}} \cos (x-\pi / 4)$ and $0<\Delta<E_{0}$, it is possible to show using Eq. (5) that for polygonal billiards [11]

$$
\int d \varphi \sum e^{\lambda_{n}(\varphi) t} \simeq 2 \pi N b_{0}+2 \pi N \sum_{n} f\left(\sqrt{E_{n}} l\right) .
$$

where $\left\{E_{n}\right\}$ are the Neumann eigenvalues of the system and $b_{0}$ is a constant [11, 12]. Thus $\lambda_{n}(\varphi)=$ $i \sqrt{E_{n}} \sin (\varphi)[13$. This is the central result of [6] when the Maslov phases are zero.

For integrable polygons, Eq. (7) can in fact be derived directly starting from Eq. (1). The eigenfunctions, $\left\{\phi_{n}\left(\theta_{1}, \theta_{2}\right)\right\}$, on the torus are such that $\phi_{n}\left(\theta_{1}^{t}, \theta_{2}^{t}\right)=$ $\Lambda_{n}(t) \phi_{n}\left(\theta_{1}, \theta_{2}\right)$ where $\Lambda_{n}(t)=e^{i \alpha_{n} t}$. On demanding that $\phi_{n}\left(\theta_{1}, \theta_{2}\right)$ be a single valued function of $\left(\theta_{1}, \theta_{2}\right)$, it follows that $\phi_{\mathbf{n}}\left(\theta_{1}, \theta_{2}\right)=e^{i\left(n_{1} \theta_{1}+n_{2} \theta_{2}\right)}$ where $\mathbf{n}=\left(n_{1}, n_{2}\right)$ is a point on the integer lattice. Thus the eigenvalue, $\Lambda_{\mathbf{n}}(t)=\exp \left\{i t\left(n_{1} \omega_{1}+n_{2} \omega_{2}\right)\right\}$.

To illustrate the relationship between $\int d \varphi \sum e^{\lambda_{n}(\varphi) t}$ and $\left\{E_{n}\right\}$, consider a rectangular billiard for which the Hamiltonian expressed in terms of the actions, $I_{1}, I_{2}$ is
$H\left(I_{1}, I_{2}\right)=\pi^{2}\left(I_{1}^{2} / L_{1}^{2}+I_{2}^{2} / L_{2}^{2}\right)$ where $L_{1}, L_{2}$ are the lengths of the two sides. With $I_{1}=\sqrt{E} L_{1} \cos (\varphi) / \pi$ and $I_{2}=\sqrt{E} L_{2} \sin (\varphi) / \pi$, it is easy to see that at a given energy, $E$, each torus is parameterized by a particular value of $\varphi$. Thus

$$
\Lambda_{\mathbf{n}}(t ; \varphi)=e^{i 2 \pi t \sqrt{E}\left(n_{1} \cos (\varphi) / L_{1}+n_{2} \sin (\varphi) / L_{2}\right)}
$$

and

$$
\begin{aligned}
\int d \varphi \sum e^{\lambda_{n}(\varphi) t} & =\sum_{\mathbf{n}} \int_{-\pi-\mu_{n}}^{\pi-\mu_{n}} d \varphi e^{i l \sqrt{E_{\mathbf{n}}} \sin \left(\varphi+\mu_{\mathbf{n}}\right)} \\
& =2 \pi \sum_{\mathbf{n}} J_{0}\left(\sqrt{E_{\mathbf{n}}} l\right)
\end{aligned}
$$

where $l=2 t \sqrt{E}, \tan \left(\mu_{\mathbf{n}}\right)=n_{1} L_{2} /\left(n_{2} L_{1}\right)$ and $E_{n}=$ $\pi^{2}\left(n_{1}^{2} / L_{1}^{2}+n_{2}^{2} / L_{2}^{2}\right)$. On separating out $\mathbf{n}=(0,0)$ from the rest, restricting the summation to the first quadrant of the integer lattice and noting that for a rectangle $b_{0}=$ 1/4, Eq. (7) follows.

The classical evolution operator thus serves to determine the Neumann spectrum in polygonal billiards. Appropriate modifications however need to be made for the Dirichlet spectrum or for systems that have caustics (the circle billiard is an example) and the construction of the evolution operator is then guided by the nature of the semiclassical trace formula (Eq. 5).

The quasiclassical evolution operator, $\mathcal{L}_{q c}$, linking the classical eigenvalues to the the desired semiclassical eigenvalues can be defined as

$$
\mathcal{L}_{q c}^{t}(\varphi) \circ \phi(\mathbf{q})=\int d \mathbf{q}^{\prime} \delta\left(\mathbf{q}-\mathbf{q}^{\prime t}(\varphi)\right) e^{-i n(t) \pi-i \nu(t) \frac{\pi}{2}} \phi\left(\mathbf{q}^{\prime}\right)
$$

where $\nu(t)=\nu\left(\mathbf{q}^{\prime t}(\varphi)\right)$ and $n(t)=n\left(\mathbf{q}^{\prime t}(\varphi)\right)$ count respectively the number of caustics and (phase altering) reflections encountered by the trajectory $\mathbf{q}^{\prime t}(\varphi)$ in time $t$. As before, the multiplicative nature of $\mathcal{L}_{q c}^{t}(\varphi)$ implies that its spectrum is of the form $\left\{e^{\lambda_{n}(\varphi) t}\right\}$ and it remains to be shown that for the quasiclassical operator defined in Eq. (10), $\left\{\lambda_{n}\right\}$ has a one-to-one correspondence with the appropriate quantum spectrum.

As before, we shall evaluate

$$
\begin{aligned}
\int d \varphi \operatorname{Tr} \mathcal{L}_{q c}^{t} & =\int_{n} d \varphi \sum e^{\lambda_{n}(\varphi) t} \\
& =\int d \varphi \int d \mathbf{q} \delta\left(\mathbf{q}-\mathbf{q}^{t}(\varphi)\right) e^{-i n(t) \pi-i \nu(t) \frac{\pi}{2}}
\end{aligned}
$$

The trajectories that contribute are once more periodic due to the delta function in the kernel. The $\mathbf{q}$ and $\varphi$ integrations can be performed along similar lines and we finally have

$$
\int d \varphi \operatorname{Tr} \mathcal{L}_{q c}^{t} \simeq N \sum_{p} \sum_{r=1}^{\infty} \frac{a_{p}}{r l_{p}} \delta\left(l-r l_{p}\right) e^{-i r n_{p} \pi-i r \nu_{p} \frac{\pi}{2}}
$$


Starting with the function $\sum_{n} g\left(\sqrt{E_{n}} l\right) \exp \left(-\beta E_{n}\right)$, it follows from Eq. (5) that for $\beta \rightarrow 0^{+}$,

$$
\int d \varphi \operatorname{Tr} \mathcal{L}_{q c}^{t}=\sum_{n} \Lambda_{n}(t)=2 \pi N C+2 \pi N \sum_{n} g\left(\sqrt{E_{n}} l\right)
$$

where $\left\{E_{n}\right\}$ now refers to the desired quantum spectrum, $g(x)=\sqrt{2 /(\pi x)} \exp (-i x+\pi / 4)$ and $C$ is a constant. Since

$$
g(x) \simeq \frac{1}{\pi} \int_{0}^{2 \pi} e^{-i x \sin (\varphi)} d \varphi
$$

for large $x$, it follows that for $v=1$,

$$
\lambda_{n}(\varphi)=i \sqrt{E_{n}} \sin (\varphi)
$$

Eq. (15) forms the central result of [6].

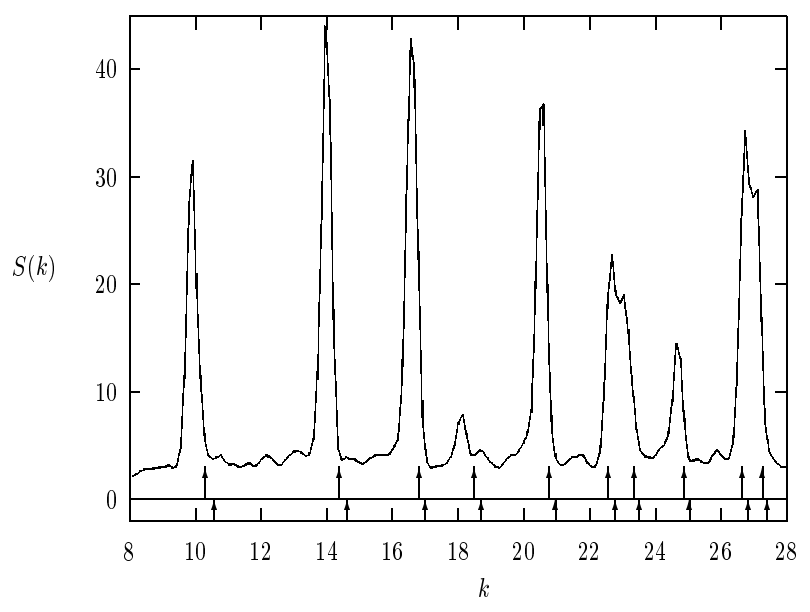

FIG. 3. The power spectrum, $S(k)$ for the triangular enclosure with base length unity. The top set of arrows mark the first ten Dirichlet eigenvalues of the triangle. The bottom set are obtained from Bogomolny's transfer operator method [14. Note that the non-zero cell size $(\epsilon)$ corresponds to the hat-function kernel rather than the delta function kernel used so far. For more details, see text.

To demonstrate this result, we shall consider a triangle with base angles $(\pi / 5,3 \pi / 10)$ and evaluate the power spectrum, $S(k)$, of the (phase weighted) fraction of trajectories averaged over 150 cells of radius $\epsilon=0.03$. The $\varphi$ integration is performed using 300 trajectories each of which has a length $2048 \times 0.025$. The result is plotted in fig. 3. The peak positions of $S(k)$ approximate the exact quantum eigenvalues well. Note however that the cells have a non-zero value of $\epsilon$ and the power spectrum depends sensitively on the choice of this quantity. In the following section therefore, we shall develop an appropriate theory that takes into account the non-zero $\epsilon$ requirement.

\section{ARBITRARY TRAJECTORY QUANTIZATION : MODIFICATIONS}

As pointed out, the existing theory does not require the $\epsilon$ neighbourhood depicted in fig. 1. All one needs is the expansion of the kernel

$$
K\left(\mathbf{q}, \mathbf{q}^{\prime t}(\varphi)\right)=\sum_{n} \phi_{n}(\mathbf{q}) \phi_{n}^{*}\left(\mathbf{q}^{\prime}\right) e^{i \sqrt{E_{n}} \sin (\varphi) t}
$$

where $\phi_{n}$ are the eigenfunctions of $\mathcal{L}_{q c}^{t}$. The power spectrum, $S(k)$, of $\int d \varphi K\left(\mathbf{q}, \mathbf{q}^{\prime t}(\varphi)\right)$ then has peaks at $\sqrt{E_{n}}$. In practice, this implies that $\epsilon$ must tend to zero since the kernel has a delta function. The results however get worse as $\epsilon$ is reduced to zero. A non-zero cell size on the other hand gives satisfactory results but is equivalent to a smoothened kernel [15]. There is thus a gap in the present understanding and it is necessary to take a fresh look at the theoretical framework.

The trouble in effect lies with the semiclassical trace formula (Eq. (5) which neglects the contributions of closed almost-periodic trajectories. In case of a polygon, these occur in families with the same symbolic dynamics and across which the action varies slowly. To see this, consider an arbitrary triangle $T$. In its immediate neighbourhood (obtained by changing the angles slightly), there exists an infinity of triangles $\left\{T^{(i)}\right\}$, each with a distinct periodic orbit spectrum but having the same symbol sequence (in which the sides are visited) for times that depend on the differences in angles. Assume now that there exists a periodic orbit corresponding to a sequence $S_{k}$ for the triangle $T^{(j)}$. Then, for all other triangles in its neighbourhood, this sequence contributes to the semiclassical trace formula, an amount (nearly) equal to the periodic orbit contribution of $T^{(j)}$ provided $\pi w_{i} \varphi_{e}^{(i)}<<\lambda$ [3]. Here $\Delta \theta_{i}$ is the angle between the initial and final momentum of the orbit, $w_{i}$ is the transverse extent of the family and $\lambda$ is the de Broglie wavelength. Thus, corresponding to every periodic family in each of the triangles $\left\{T^{(i)}\right\}$, there exists an almost-periodic family in the triangle $T$ whose contribution is comparable to that of periodic orbit families in these neighbouring triangles.

The semiclassical trace formula should thus incorporate CAP orbits and the modified expression is [3] :

$$
\begin{aligned}
\rho(E) & \simeq \rho_{a v}(E)+\sum_{i} \frac{a_{i}}{\sqrt{8 \pi^{3} k l_{i}}} \\
& \times \cos \left(k l_{i}-\pi / 4\right) \frac{\sin \left(k \Delta \theta_{i} w_{i} / 2\right)}{k \Delta \theta_{i} w_{i} / 2}
\end{aligned}
$$

where the sum over $i$ runs over closed almost-periodic and periodic orbit families of transverse extent $w_{i}, l_{i}$ is the average length of such a family (taken as the length of the orbit at the centre of the band) while $\Delta \theta_{i}$ is the angle between the initial and final momentum of the orbit. Note that at any finite $k$, there exists a CAP family for which the de Broglie wavelength, $\lambda>>w_{i} \Delta \theta_{i}$ so 
that the family contributes to the modified trace formula with a weight comparable to that of periodic families $\left(\mathcal{O}\left(1 / k^{1 / 2}\right)\right)$.

The modified trace formula (Eq. 17) however fails to relate the quantum eigenvalues with the eigenvalues of $\mathcal{L}_{q c}$ since its $\delta$ function kernel chooses only periodic orbits. The kernel function must therefore be suitably smoothened to achieve such a correspondence.

In order that there exists a direct relationship between the eigenvalues of a quasiclassical operator, $\tilde{\mathcal{L}}_{q c}$ and the quantum eigenvalues, $\left\{E_{n}\right\}$, the kernel function corresponding to $\tilde{\mathcal{L}}_{q c}$ must be [16]

$$
\tilde{K}_{q c}\left(\mathbf{q}, \mathbf{q}^{\prime}, t\right)=K_{s}\left(\mathbf{q}, \mathbf{q}^{\prime}, t\right) e^{-i n(t) \pi-i \nu(t) \frac{\pi}{2}}
$$

where

$$
K_{s}\left(\mathbf{q}, \mathbf{q}^{\prime}, t\right)=\frac{\sin \left(\tilde{k}\left(q_{\|}-q_{\|}^{\prime t}(\varphi)\right)\right.}{\pi\left(q_{\|}-q_{\|}^{\prime t}(\varphi)\right)} \frac{\sin \left(\tilde{k}\left(q_{\perp}-{q_{\perp}^{\prime}}^{t}(\varphi)\right)\right.}{\pi\left(q_{\perp}-{q_{\perp}^{\prime}}^{t}(\varphi)\right)} .
$$

Note that $\lim _{\tilde{k} \rightarrow \infty} K_{s}\left(\mathbf{q}, \mathbf{q}^{\prime}, t\right)=\delta\left(\mathbf{q}-\mathbf{q}^{\prime t}(\varphi)\right)$. The subscript $s$ thus denotes the smoothened kernel. As before, the multiplicative nature of $\tilde{\mathcal{L}}_{q c}^{t}(\varphi)$ implies that its spectrum is of the form $\left\{e^{\lambda_{n}^{s}(\varphi) t}\right\}$ and we shall now show that for the quasiclassical operator defined in Eq. 18, $\left\{\lambda_{n}^{s}\right\}$ has a one-to-one correspondence with the appropriate quantum spectrum.

Note that in general, $q_{\|}-q_{\|}{ }^{t}(\varphi)=l_{i}+q_{\perp} \Delta \theta_{i}-l=\Delta q_{\|}$ for the $i^{\text {th }}$ (CAP or periodic) family while $q_{\perp}-q_{\perp}{ }^{t}(\varphi)=$ $l_{i} \varphi=\Delta q_{\perp}$ so that

$$
\int d \varphi d \mathbf{q} K_{s}(\mathbf{q}, \mathbf{q}, t)=\int d \varphi d q_{\|} d q_{\perp} \frac{\sin \left(\tilde{k} \Delta q_{\|}\right)}{\pi \Delta q_{\|}} \frac{\sin \left(\tilde{k} \Delta q_{\perp}\right)}{\pi \Delta q_{\perp}}
$$

A few approximations are now in order to keep the derivation simple. First, we shall replace $\Delta q_{\|}$in the denominator by its mean value (at $q_{\perp}=0$ ) so that $\Delta q_{\|} \simeq l-l_{i}$ Thus

$$
\int_{-w_{i} / 2}^{w_{i} / 2} d q_{\perp} \frac{\sin \left(\tilde{k} \Delta q_{\|}\right)}{\pi \Delta q_{\|}} \simeq 2 \frac{\sin \left(\tilde{k} \Delta \theta_{i} w_{i} / 2\right)}{\tilde{k} \Delta \theta_{i}} \delta_{\tilde{k}}\left(l-l_{i}\right)
$$

where $\delta_{\tilde{k}}\left(l-l_{i}\right)=\sin \left(\tilde{k}\left(l-l_{i}\right)\right) /\left(\pi\left(l-l_{i}\right)\right)$. We shall next consider $\tilde{k}$ finite but sufficiently large so that

$$
\int d \varphi \frac{\sin \left(\tilde{k} \Delta q_{\perp}\right)}{\pi \Delta q_{\perp}} \simeq \frac{1}{l_{i}}
$$

The $q_{\|}$integration needs no approximation and yields $\int d q_{\|}=l_{i}^{p}$ where $l_{i}^{p}$ refers to the length of the orbit (primitive when $\left.\Delta \theta_{i}=0\right)$. Finally then,

$$
\int d \varphi \operatorname{Tr} \tilde{\mathcal{L}}_{q c}^{t} \simeq \sum_{i} N \frac{a_{i}}{l_{i}} \beta_{i} \delta_{\tilde{k}}\left(l-l_{i}\right) e^{-i n_{i} \pi-i \nu_{i} \frac{\pi}{2}}
$$

where $\beta_{i}=\sin \left(\tilde{k} \Delta \theta_{i} w_{i} / 2\right) /\left(\tilde{k} \Delta \theta_{i} w_{i} / 2\right)$ and $a_{i}=w_{i} l_{i}^{p}$. For a polygon, $n_{i}$ is even for both CAP and periodic families while $\nu_{i}=0$. Thus as $\tilde{k} \rightarrow \infty$, Eq. (23) reduces to Eq. (4). As before, we have neglected the contribution of isolated orbits from both the classical and semiclassical trace formulae.

The eigenvalues of $\tilde{\mathcal{L}}_{a c}^{t}$ can be related to quantum eigenvalues using Eqns. (23) and (17) and we merely state the final result :

$$
\lambda_{n}^{s}(\varphi)=\imath \sqrt{E_{n}} \sin (\varphi)
$$

This is no different from our earlier result but in practical terms, the use of the smoothened kernel justifies the use of the $\epsilon$ neighbourhood since $K_{s}$ contributes substantially only in a small neighbourhood around $\mathbf{q}$, the size of which is determined by $\tilde{k}$. Note that the correspondence demands that the quantity $\tilde{k}$ in $K_{s}$ be identified with $\sqrt{E_{\max }}$ where $E_{\max }$ is the maximum energy that one is interested in. Thus $\tilde{k}$ also determines the time increment in the evolution of the kernel.

Undoubtedly, several other smoothened kernels are just as appropriate. For instance, if one evaluates the fraction of trajectories in an $\epsilon$ neighbourhood of $\mathbf{q}$, the hat function of width $\epsilon$ and height $1 / \epsilon$ is the appropriate kernel to use. The role of $\tilde{k}$ is then replaced by $\frac{1}{\epsilon}$. On the other hand, if one chooses a Gaussian centred at $\mathbf{q}$, the appropriate smoothened kernel is

$$
K_{s}^{G}\left(\mathbf{q}, \mathbf{q}^{\prime}, t\right)=\frac{e^{-\frac{\left(q_{\|}-q_{\|}^{\prime}{ }^{t}(\varphi)\right)^{2}}{2 \sigma^{2}}}}{\sqrt{2 \pi \sigma^{2}}} \frac{e^{-\frac{\left(q_{\perp}-q_{\perp}^{\prime}{ }^{t}(\varphi)\right)^{2}}{2 \sigma^{2}}}}{\sqrt{2 \pi \sigma^{2}}}
$$

On performing the three $\left(\varphi, q_{\|}, q_{\perp}\right)$ integrals similarly, it is possible to show that

$$
\int d \varphi \operatorname{Tr} K_{s}^{G}=\sum_{i} \frac{a_{i}}{l_{i}}\left\{1-\frac{1}{3}\left(\frac{w_{i} \Delta \theta_{i}}{2^{3 / 2} \sigma}\right)^{2}+\ldots\right\} \frac{e^{-\frac{\left(l-l_{i}\right)^{2}}{2 \sigma^{2}}}}{\sqrt{2 \pi \sigma^{2}}}
$$

which approximates Eq. (23) for orbits with $\Delta \theta_{i}$ small provided $1 / \sigma$ is identified with $\tilde{k}$. Thus any smoothened delta function ought to work reasonably well.

Finally, a few remarks about the neglect of the isolated periodic orbits are in order. First, these are finite in number and have a smaller contribution in both the (quasi)classical as well as the semiclassical trace formula compared to periodic and almost-periodic families. Then again, diffractive contributions [17] can be of the same order as isolated periodic orbit contributions in the semiclassical trace formula. Thus, in order to be able to compare the semiclassical eigenvalues and the eigenvalues of $\tilde{\mathcal{L}}_{q c}$, we have consistently neglected isolated orbits.

We provide some numerical results now. We first evaluate the function $G(t)=\int d \varphi \tilde{K}_{q c}$ for the $(199 \pi / 1011,31 \pi / 103)$ triangle of unit base length using about 10000 trajectories for the $\varphi$ integration. To achieve smoothening, we use the Hanning window function together with a Gaussian damping and find the 
power spectrum, $S(k)$, of $G(t) e^{-\beta t^{2}}$. This is the intensity weighted spectrum, $\sum_{n}\left|\phi_{n}(\mathbf{q})\right|^{2} \delta_{s}\left(k-k_{n}\right.$ ) (where $\delta_{s}$ is a smoothened delta function), for a single pair of points $\left(\mathbf{q}, \mathbf{q}^{\prime}=\mathbf{q}\right)$. Thus, the number of distinct and unambiguous peaks in $S(k)$ depends on the value of $\tilde{k}$ and $\left|\phi_{n}(\mathbf{q})\right|^{2}$ so that by changing $\mathbf{q}$, a different set of

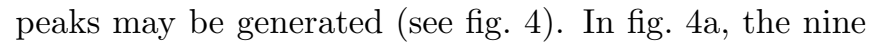
highest peak locations (those above the dashed line) are compared with the exact quantum eigenenergies while in fig. 4lb, the highest five are compared. In all cases, the agreement is good and the slight difference between peak locations and the exact quantum levels is due to the semiclassical nature of the calculation.
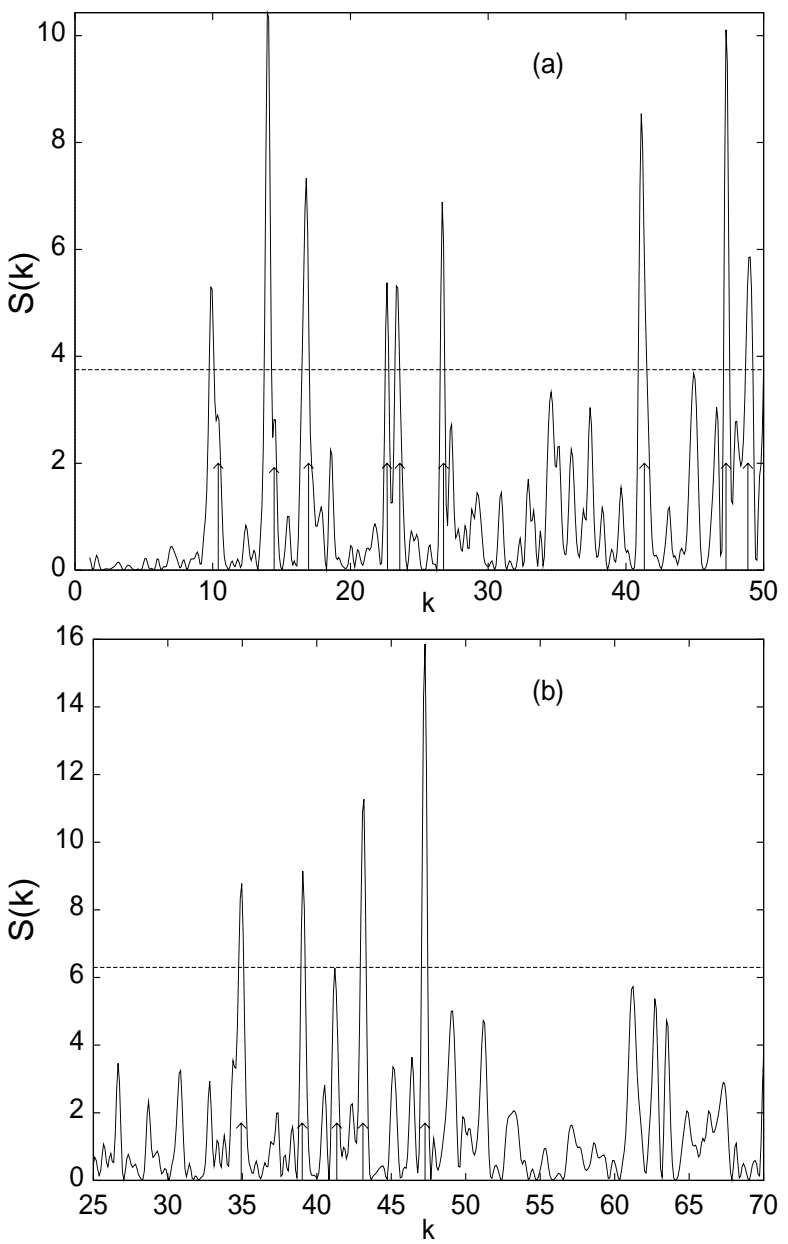

FIG. 4. The power spectrum $S(k)$ of $G(t) e^{-\beta t^{2}}$ as a function of $k$ is shown for two different sets of points $\left(\mathbf{q}, \mathbf{q}^{\prime}=\mathbf{q}\right)$ in (a) and (b) respectively. The arrows in (a) mark the exact quantum eigenenergies corresponding to the nine tallest peaks while those in (b) mark the five tallest peaks. The dashed lines are used to mark the demarcate the tallest peaks in both cases. Here $\tilde{k}=100, \beta=0.01$ and the length of each trajectory is $2048 \pi / \tilde{k}$. There exist 37 eigenvalues for $k<50$.

The intensity weighted spectrum of fig. 4 thus demonstrates that arbitrary trajectories can be used to extract information about the quantum eigenstates. Can this however be used as an effective quantization method to determine all eigenvalues? Fig. 3 indicates that this may indeed be possible. A natural way of achieving this is by integrating the intensity weighted spectrum over $\mathbf{q}[18]$. In practice, only a few q values (typically 10-20) provide information of about $80 \%$ of the eigenvalues in a given energy range.

\section{DISCUSSION AND CONCLUSIONS}

It is worth recalling the sources of error in the quantization recipe presented here. The theoretical basis presented in sections [II and III clearly indicates that the eigenvalues obtained using this method are at best "semiclassical" in nature since the modified trace formula, which connects the eigenvalues of $\tilde{\mathcal{L}}_{q c}$ with the quantum eigenvalues, is only approximate as higher order corrections $(\mathcal{O}(1 / k)$ due to isolated and diffractive orbits) have been neglected. In cases when the corrections are zero (such as in the rectangular or equilateral billiards), the ATQM does give exact results. However, there are examples of other integrable billiards (such as the circle billiard) when corrections to the semiclassical trace formula are non-zero and the ATQM gives only the EBK eigenvalues [19].

There can however be a further source of error in the approximation $N_{p} \simeq N$ (see the discussion after Eq. 目) especially in non-generic systems where a significant fraction of long periodic orbits access fewer momentum directions than the permissible number. However, we believe that in generic situations, the approximation is fair.

Finally, since a comparison of this method with periodic orbit theory is inevitable, it must be reiterated that the "usual" periodic orbit theory neglects closed almostperiodic orbits and hence cannot give correct results in generic situations. The modified periodic orbit theory (or trace formula) does include CAP orbits. However, due to difficulties in enumerating them, energy domain quantization is expected to be quite cumbersome while the ATQM scores well due to its simplicity. There are non-generic situations however where closed almost periodic trajectories do not contribute significantly and this occurs in systems where the number of momentum directions accessible is small and $\Delta \theta_{i}$ is therefore always "large". In such situations, diffraction effects assume greater significance and the ATQM may not be as effective.

In summary, we have provided a simple algorithm for determining the intensity weighted semiclassical spectrum of polygonal billiards and shown that a smoothened delta function kernel for the quasiclassical evolution of densities is necessary at finite energies to incorporate the effects of closed almost-periodic orbits. 
[1] M. C. Gutzwiller, Chaos in Classical and Quantum Mechanics (Springer Verlag, New York, 1990); in Chaos and Quantum Physics, Les Houches 1989, eds. M.J.Giannoni, A.Voros and J.Zinn-Justin, North Holland, 1991.

[2] H. Primack and U. Smilansky, J. Phys. A 27, 4439 (1994).

[3] D. Biswas, Phys. Rev. E 61, 5129 (2000).

[4] These orbits close in position but the angle, $\Delta \theta$, between the initial and final momentum (at the point where the orbit closes) is non-zero but small. In contrast, $\Delta \theta=0$ for periodic families.

[5] E. J. Heller, J. Chem. Phys. 68, 3891 (1978); J. Chem. Phys. 94, 2723 (1991); S. Tomsovic and E. J. Heller, Phys. Rev. Lett. 67, 664 (1991).

[6] D. Biswas, chao-dyn/9804013 \& in Nonlinear Dynamics and Computational Physics, ed. V. B. Sheorey, Narosa, New Delhi, 1999.

[7] See also D. Biswas and S. Sinha, Phys. Rev. Lett. 71, 3790 (1993) for an application to a chaotic system.

[8] The term quasiclassical is used here to distinguish the ATQM from the semiclassical trace formula.

[9] See for instance Y. Shimizu and A. Shudo, Chaos, Solitons and Fractals, 5, 1337 (1995) for periodic orbit quantization of polygons.

[10] For the circle billiard, $\varphi$ is related to the angular momentum rather than the linear momentum.

[11] D.Biswas and S.Sinha, Phys. Rev. Lett. 70, 916 (1993).

[12] D.Biswas, Phys. Rev. E54, R1044 (1996).

[13] The function $f(x)$ is the asymptotic expansion of $J_{0}(x)$.

[14] E.B.Bogomolny, Nonlinearity 5, 805 (1992).

[15] The cell size, $\epsilon$, is arbitrary at this point and appears to be a free parameter. We shall relate $\epsilon$ to $E_{\max }$ where $E_{\text {max }}$ is the maximum energy that one is interested in.

[16] The exact choice of the kernel is dictated by the modified semiclassical trace formula. There are however other options as discussed in the text.

[17] E. Bogomolny, N. Pavloff and C. Schmit, chao-dyn/9910037.

[18] Averaging over $q$ as in fig. 3 is a crude way of extracting this information.

[19] J. B. Keller and S. I. Rubinow, Ann. Phys. (N.Y.) 9, 24 (1960). 